\title{
Cellular characterisation of the GCKR P446L variant associated with type 2 diabetes risk
}

\author{
M. G. Rees • S. Wincovitch $•$ J. Schultz • \\ R. Waterstradt • N. L. Beer $\cdot$ S. Baltrusch • \\ F. S. Collins • A. L. Gloyn
}

Received: 26 August 2011 / Accepted: 28 September 2011 /Published online: 25 October 2011

(C) Springer-Verlag 2011

\begin{abstract}
Aims/hypothesis Translation of genetic association signals into molecular mechanisms for diabetes has been slow. The glucokinase regulatory protein (GKRP; gene symbol $G C K R) \mathrm{P} 446 \mathrm{~L}$ variant, associated with inverse modulation of glucose- and lipid-related traits, has been shown to alter the kinetics of glucokinase (GCK) inhibition. As GCK inhibition is associated with nuclear sequestration, we aimed to determine whether this variant also alters the direct interaction between GKRP and GCK and their intracellular localisation.

Methods Fluorescently tagged rat and human wild-type (WT)- or P446L-GCKR and GCK were transiently transfected into HeLa cells and mouse primary hepatocytes. Whole-cell and nuclear fluorescence was quantified in individual cells exposed to low- or high-glucose conditions (5.5 or $25 \mathrm{mmol} / \mathrm{l}$ glucose, respectively). Interaction between GCK and GKRP was measured by sensitised emission-based fluorescence resonance energy transfer (FRET) efficiency.
\end{abstract}

\footnotetext{
M. G. Rees $・$ N. L. Beer $\cdot$ A. L. Gloyn $(\bowtie)$

Oxford Centre for Diabetes Endocrinology \& Metabolism,

University of Oxford, Churchill Hospital,

Headington,

Oxford OX3 7LJ, UK

e-mail: anna.gloyn@drl.ox.ac.uk

M. G. Rees $\cdot$ S. Wincovitch $\cdot$ F. S. Collins

National Human Genome Research Institute,

National Institutes of Health,

Bethesda, MD, USA

J. Schultz $\cdot$ R. Waterstradt $\cdot$ S. Baltrusch

Institute for Medical Biochemistry \& Molecular Biology,

University of Rostock,

Rostock, Germany

Results P446L-GKRP had a decreased degree of nuclear localisation, ability to sequester GCK and direct interaction with GCK as measured by FRET compared with WTGKRP. Decreased interaction was observed between WTGKRP and GCK at high compared with low glucose, but not between P446L-GKRP and GCK. Rat WT-GKRP and P446L-GKRP behaved quite differently: both variants responded to high glucose by diminished sequestration of GCK but showed no effect of the P446L variant on nuclear localisation or GCK sequestration.

Conclusions/interpretation Our study suggests the common human P446L-GKRP variant protein results in elevated hepatic glucose uptake and disposal by increasing active cytosolic GCK. This would increase hepatic lipid biosynthesis but decrease fasting plasma glucose concentrations and provides a potential mechanism for the protective effect of this allele on type 2 diabetes risk.

Keywords Association study - Genetics · Glucokinase . Molecular mechanism

\begin{tabular}{ll}
\multicolumn{2}{l}{ Abbreviations } \\
GKRP & Glucokinase regulatory protein \\
hGKRP & Human GKRP \\
rGKRP & Rat GKRP \\
GCK & Glucokinase \\
ECFP & Enhanced cyan fluorescent protein \\
EGFP & Enhanced green fluorescent protein \\
EYFP & Enhanced yellow fluorescent protein \\
FRET & Fluorescence resonance energy transfer \\
FRETN & Sensitised emission-based FRET efficiency \\
F6P & Fructose 6-phosphate \\
F1P & Fructose 1-phosphate \\
WT & Wild-type \\
SNP & Single nucleotide polymorphism
\end{tabular}




\section{Introduction}

The past 4 years have seen a rapid expansion in the number of reproducibly associated loci for type 2 diabetes and related traits. However, progress in translating these genetic associations into biological mechanisms for disease has been slow [1,2]. Contributing factors have included small effect sizes and the mapping of association signals to poorly annotated, often intergenic, sequences [3]. Signals that include an obvious (e.g. non-synonymous) variant in a biologically plausible gene provide tractable targets for functional follow-up and a framework for exploring approaches to variant characterisation [2].

Glucokinase (GCK) regulates glucose storage and disposal in the liver. Hepatic GCK activity is modulated by glucokinase regulatory protein (GKRP; gene symbol GCKR), a competitive inhibitor of GCK. Fructose 6phosphate (F6P) and fructose 1-phosphate (F1P) enhance and reduce GKRP-mediated inhibition, respectively $[4,5]$. A further layer of regulation exists in the form of cellular localisation: GKRP sequesters GCK to the nucleus at low glucose concentrations, further inhibiting GCK activity and glycolysis [6]. At higher glucose concentrations, glucosemediated dissociation of GCK from GKRP activates GCK and exposes its nuclear export signal [7, 8].

A common GCKR single nucleotide polymorphism (SNP) (rs1260326 [c.1337C>T; p.P446L]) has been reproducibly associated with an inverse relationship between glucose and triacylglycerol levels [9]. This seemingly paradoxical relationship also extends to other glucose- and lipid-related traits. The glucose-lowering leucine allele is also associated with decreased fasting insulin, HOMAestimated insulin resistance and reduction of type 2 diabetes risk [10] but increased triacylglycerol and total cholesterol levels [11]. This variant is one of only a handful of candidate non-synonymous variants identified to date from type 2 diabetes association studies [2, 12]. We have previously shown that variant P446L-GKRP increases GCK activity as a result of diminished regulation by F6P and have proposed that this results in increased hepatic glycolytic flux and de novo lipogenesis, accounting for the observed associated phenotypes [12]. Interestingly, this kinetic effect was not seen with rat P446L-GKRP [13], likely due to species differences in regulation by F6P [14].

However, kinetic mechanisms alone are not sufficient to explain GKRP regulation of GCK in vivo. For example, $G c k r$-knockout mice display impaired postprandial glucose handling and paradoxical reductions in GCK protein levels and activity in spite of the removal of GKRP inhibition [15, 16]. This suggests that nuclear interaction with GKRP plays an important role in regulating GCK protein concentration and stability and is essential for the maintenance of glucose homeostasis. In addition, the P446L variant is associated with inverse modulation of fasting and $2 \mathrm{~h}$ glucose levels, an observation not readily explained by kinetic data [10]. As the contribution of GKRP and GCK cellular localisation to phenotypes associated with the P446L-GKRP variant protein is currently unexplored, the aims of the current study were to assess the impact of this variant on the ability of GKRP to sequester GCK in the nucleus and to further explore potential differences between rat and human regulatory proteins.

\section{Methods}

Cloning of fluorescent fusion plasmids Human GCK (Mammalian Gene Collection; ATCC, Manassas, VA, USA) was amplified using primers containing BamHI and HindIII restriction sites and subcloned into the pEGFP-c3 vector encoding enhanced green fluorescent protein (EGFP). Generation of enhanced cyan fluorescent protein (ECFP)-tagged $G C K$ (pECFP-GCK) has been described by Baltrusch et al. [17]. PCR primers containing Eco47III and EcoRI sites were used to amplify human GCKR (Mammalian Gene Collection) and replace rat Gckr in pEYFP-N1GCKR [17]. The P446L mutation was introduced into rat and human pEYFP-N1-GCKR plasmids by PCR-based sitedirected mutagenesis (Agilent Biotechnologies, Santa Clara, CA, USA). The enhanced yellow fluorescent protein (EYFP) in both rat and human pEYFP-N1-GCKR [17] was replaced by mCherry [18] using AgeI and BsrGI restriction sites. Two independent preparations of all plasmids were generated and verified by sequencing. Primer sequences are available upon request.

Cell culture and transient transfection of HeLa cells HeLa cells were grown in DMEM with 5.5 or $25 \mathrm{mmol} / 1$ glucose supplemented with $10 \%$ (vol./vol.) dialysed fetal bovine serum and penicillin/streptomycin (all Invitrogen, Carlsbad, CA, USA). For quantitative fluorescence microscopy, $4 \times 10^{4}$ cells were seeded into four-chamber slides (BD Biosciences, San Jose, CA, USA) and cultured for $24 \mathrm{~h}$. Co-transfection of mCherry-GCKR and EGFP-GCK was initially carried out with a range of plasmid DNA concentrations at ten separate $G C K / G C K R$ molar ratios between 40:1 and 1:40. Nuclear localisation of GCK was observed for $G C K / G C K R$ ratios between 1:1 and 1:40. A ratio of 1:4 was used for all future experiments as this is in agreement with the published $G C K / G C K R$ mRNA ratio in liver [12]. Optimal transfection efficiency and fluorescence intensity was observed with transfection of $100 \mathrm{ng}$ total DNA and $0.75 \mu \mathrm{l}$ of Lipofectamine 2000 (Invitrogen). Transfection was carried out according to the manufacturer's instructions at $5.5 \mathrm{mmol} / \mathrm{l}$ glucose. The medium was replaced $5 \mathrm{~h}$ after transfection. 
Slides were incubated at 5.5 or $25 \mathrm{mmol} / \mathrm{l}$ glucose at $22 \mathrm{~h}$ post-transfection and fixed with formaldehyde $24 \mathrm{~h}$ posttransfection for mounting with VectaShield Mounting Medium with DAPI (Vector Laboratories, Burlingame, CA, USA). Each preparation condition was transfected two times. For fluorescence resonance energy transfer (FRET) studies, cells were seeded at a density of $2 \times 10^{5}$ cells on $35 \mathrm{~mm}$ glass-bottom dishes (MatTek, Ashland, MA, USA) and grown for $24 \mathrm{~h}$. Thereafter, cells were transfected with $1 \mu \mathrm{g}$ DNA (1:1 ratio of the EYFP and ECFP plasmid) and $2 \mu \mathrm{l}$ jetPEI (Qbiogene, Montreal, QC, Canada) according to the manufacturer's instructions. The culture medium was replaced 1 day after transfection, and the cells were incubated for another $24 \mathrm{~h}$ with medium containing 5.5 or $25 \mathrm{mmol} / \mathrm{l}$ glucose.

Hepatocyte isolation, culture, and transient transfection Primary hepatocytes were isolated from C57/BL6 mice using a modification of the collagenase perifusion methods of Seglen [19] and Bader et al. [20]. Isolated cells were suspended in Williams' medium E supplemented with $10 \mathrm{mmol} / \mathrm{l}$ glucose, $5 \%$ (vol./vol.) fetal calf serum, $1 \times 10^{-4} \mathrm{mmol} / 1$ dexamethasone, and $1 \times 10^{4} \mathrm{pmol} / \mathrm{l}$ insulin. Hepatocytes were seeded at a density of $4 \times 10^{5}$ cells on $35 \mathrm{~mm}$ collagen-coated glassbottom dishes (MatTek, Ashland, MA, USA) or on 12-well plates and incubated for $24 \mathrm{~h}$ in a humidified atmosphere at $37^{\circ} \mathrm{C}$ and $5 \% \mathrm{CO}_{2}$. Cells were transfected with $1 \mu \mathrm{g}$ DNA (either EYFP plasmid alone or 1:1 ratio of the EYFP and ECFP plasmid) and $3.2 \mu$ l PromoFectine Hepatocyte (PromoCell, Heidelberg, Germany) according to the manufacturer's instructions. Thereafter, hepatocytes were cultured for $24 \mathrm{~h}$ and finally incubated in medium with either 5.5 or $25 \mathrm{mmol} / \mathrm{l}$ glucose for $2 \mathrm{~h}$.

Fluorescence microscopy and image analysis Confocal images were acquired using a Zeiss LSM 510 NLO Meta system mounted on a Zeiss Axiovert 200M microscope with an oil-immersion Plan-Apochromat $63 \times / 1.4$ DIC objective lens. Excitation wavelengths of $488 \mathrm{~nm}(2 \%)$, $561 \mathrm{~nm}(5 \%)$ and $770 \mathrm{~nm} \mathrm{(3 \% )}$ were used for detection of EGFP-GCK, mCherry-GKRP and DAPI, respectively. Confocal images were post-processed using the MediaCybernetics Image-Pro Plus v7.0 software package (Bethesda, MD, USA) with a custom macro designed to calibrate, segment (nuclei touching the image border were not included) and outline the nuclei, outline the cell border, count and record measurements. Measurements included nucleus and cell areas (square micrometres) and fluorescent intensity (minimum, maximum, mean, standard deviation and sum). The nuclei outlines were obtained by using DAPI staining as the template and propagating that outline throughout the green and red channels. The cell border outlines were obtained using the green and red signals. Twenty independent transfection control images numbering
324 total nuclei were processed, along with 143 cotransfection images totalling 1,472 nuclei. To address the possibility that differences in localisation might be driven by differences in fluorescence intensity between variants, cells were also divided into four groups matched for either whole-cell mCherry fluorescence intensity, EGFP fluorescence intensity or the ratio of mCherry/EGFP intensity in individual cells.

For FRET experiments, a cellR/Olympus IX81 inverted microscope system (Olympus, Hamburg, Germany) equipped with a Cellcubator was used, as described by Langer et al. [21]. HC436/24-458BS-HC483/32 and HQ500/24-520BS-D542/27 single-band filter sets were used for ECFP and EYFP, respectively (AHF Analysentechnik, Tübingen, Germany). For FRET analyses, ECFP and EYFP emission were detected simultaneously using a DV-CC Dual View Simultaneous Imaging System (Optical Insights, LLC, Tucson, AZ, USA) equipped with a 505 dexr beam splitter and D472/30 and HQ542/27 emission filters (AHF Analysentechnik). For FRET analyses and calculation of the ECFP nuclear:cytoplasmic ratio, glassbottom dishes were fixed on the microscope stage and images were obtained with an UPLSAPO $60 \times 1.35$ numerical aperture oil-immersion objective (Olympus). For calculation of the EYFP nuclear to cytoplasmic ratio on 12 -well plates, an UPLSAPO $20 \times 0.75$ numerical aperture objective (Olympus) was used and all motorised devices of the microscope system were synchronised by the scanR acquisition software (Olympus). The image focus was automatically determined with a gradient method from transmitted light images. Finally, the mean fluorescence was calculated as average grey value in selected regions in the nucleus and cytoplasm in individual cells after background correction. The sensitised emission-based FRET efficiency (FRETN) was calculated from the ECFP emission with excitation at $436 \mathrm{~nm}$, EYFP emission with excitation at $436 \mathrm{~nm}$ and EYFP emission with excitation at $500 \mathrm{~nm}$, based on the calculation of Vanderklish et al. [22]. FRETN measurements were validated using an ECFPEYFP fusion construct as described by Langer et al. [21].

Statistical analysis Statistical analyses were performed by ANOVA followed by Bonferroni's test for multiple comparison or $t$ tests for independent samples using the Prism analysis program (Graphpad, San Diego, CA, USA). Data are presented as means \pm SEM. $p$ values $<0.05$ (two-tailed) were considered significant.

\section{Results}

Localisation of GKRP fluorescent fusion proteins in HeLa cells and mouse primary hepatocytes To investigate the 
subcellular localisation of human GKRP (hGKRP) and the effect of the P446L variant on this localisation, mCherrytagged GCKR fusion plasmids were transiently transfected into HeLa cells. HeLa cells were chosen as an established model known to exhibit efficient glucose uptake and lacking endogenous expression of GCKR and GCK [7, 23]. Both wild-type (WT)-hGKRP and P446L-hGKRP localised strongly to the nucleus, but an appreciable increase in cytoplasmic fluorescence intensity was detectable for P446L-hGKRP (Fig. 1a,b). To quantify this apparent difference in localisation, the ratio of nuclear to whole-cell fluorescence in individual cells was calculated. Cells with very low EGFP or mCherry fluorescence intensities $(<50,000$ arbitrary fluorescence units) were excluded from statistical analysis. However, exclusion of these data did not change the significance of any result. WT-hGKRP ( $n=20$ cells) was associated with significantly increased nuclear localisation when compared with P446LhGKRP $(n=21)(83.4 \pm 1.4 \%$ nuclear localisation compared with $65.1 \pm 3.1 \%$, mean \pm SEM; $p<0.001$; Fig. 1c). Previous studies have revealed substantial kinetic differences between human and rat GKRP (rGKRP) [14]. However, no studies have compared cellular localisation between rat and human regulatory proteins. Accordingly, we also generated and transiently transfected mCherry-tagged rat Gckr. The P446L variant was also introduced into the rat $G c k r$ sequence. There was no significant difference in the nuclear-to-whole-cell-fluorescence ratio of WT-rGKRP $(62.8 \pm 2.3 \% ; n=20)$ and P446L-rGKRP $(56.3 \pm 1.5 \%$; $n=26$; Fig. 1d). Both WT-rGKRP and P446L-rGKRP showed significantly decreased nuclear localisation compared with WT-hGKRP $(p<0.001$; ANOVA/Bonferroni correction). Localisation of all GKRPs was independent of glucose concentration ( $p>0.1$; data not shown).

To confirm our observations in HeLa cells in a physiologically relevant primary cell type, we assessed the localisation of transiently transfected human and rat GKRPs in primary mouse hepatocytes (Fig. 1d). EYFP-WT-hGKRP (nuclear/cytoplasmic ratio $5.94 \pm 0.40 ; n=45$ individual cells) was again significantly associated $(p<0.001)$ with increased nuclear localisation compared with EYFP-P446LhGKRP $(4.16 \pm 0.25 ; n=52)$, EYFP-WT-rGKRP $(3.37 \pm 0.17$; $n=36)$, and EYFP-P446L-rGKRP $(3.45 \pm 0.18 ; n=37)$. Localisation of all GKRPs was independent of glucose concentration.

Co-transfection of human fluorescent GCK and GKRP fusion plasmids in HeLa cells Transiently transfected human EGFP-GCK localised entirely to the cytoplasm of HeLa cells (Fig. 2a). In accordance with previous in vivo studies, localisation was not affected by glucose concentration (data not shown) [15]. Co-transfection of GCK and human or rat WT- and P446L-GKRP resulted in nuclear sequestration of GCK (Fig. 2b,c). Incubation for $2-16 \mathrm{~h}$ at a series of glucose concentrations (1-25 $\mathrm{mmol} / \mathrm{l})$ resulted in a mix of nuclear and cytoplasmic localisation for both GKRP and GCK (data not shown). Complete glucose-dependent export of GCK was not observed even at high glucose concentrations, consistent with previous observations in rat hepatocytes [17, 24]. Accordingly, quantitative image analysis was undertaken at two glucose concentrations ( 5.5 and $25 \mathrm{mmol} / \mathrm{l}$ glucose) which have been previously used to investigate the GCK:GKRP interaction as they are either side of the glucose $\mathrm{S}_{0.5}$ (substrate concentration needed for half maximal rate) for GCK (approximately $7.5 \mathrm{mmol} / \mathrm{l})$ [17].

Quantitative analysis was undertaken on a total of 787 cells from two transfections per glucose concentration and from two independent plasmid preparations per variant. In the presence of GCK at $5.5 \mathrm{mmol} / \mathrm{l}$ glucose, P446L-hGKRP was again associated with decreased nuclear localisation compared with WT-hGKRP (Fig. 2d; 67.4 $\pm 2.5 \%$ vs $73.6 \pm 1.8 \%$
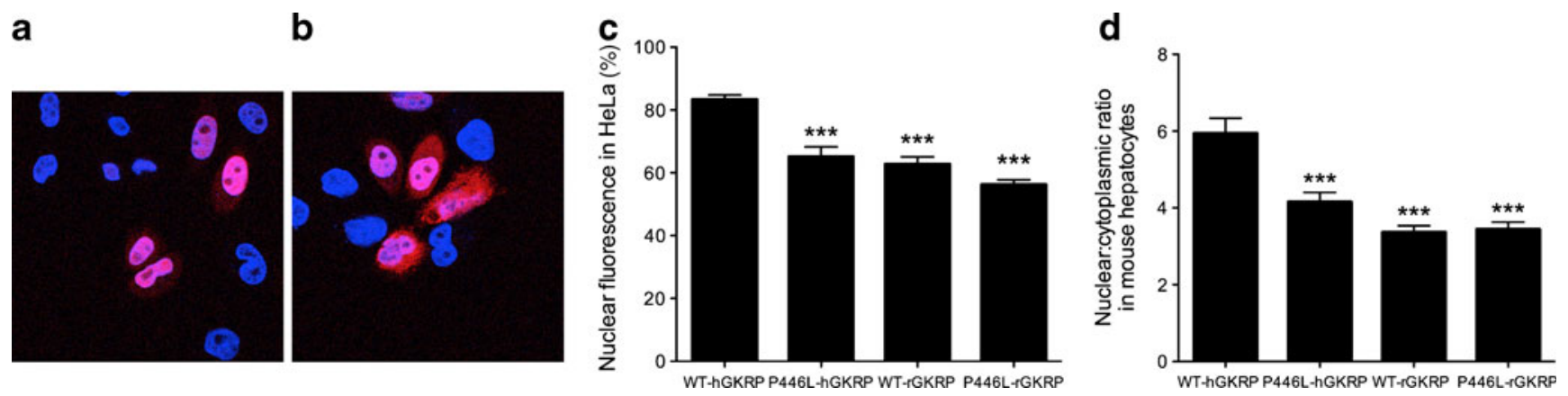

Fig. 1 Localisation of GKRP fluorescent fusion proteins at $5.5 \mathrm{mmol} / 1$ glucose. Visualisation of mCherry-WT-hGKRP (a) or P446L-hGKRP (b) and overlap with DAPI on transient transfection into HeLa cells. c Quantification of degree of nuclear fluorescence for human and rat GKRPs in HeLa cells. Data are expressed as means \pm SEM of two individual experiments with a total of 20-26 cells

analysed. d Quantification of nuclear/cytoplasmic ratio of human and rat GKRPs on transient transfection into primary mouse hepatocytes. Data are expressed as means \pm SEM of two individual experiments with a total of $36-52$ cells analysed. ${ }^{* * *} p<0.001$ compared with WT-hGKRP (ANOVA/Bonferroni correction) 
Fig. 2 Localisation of cotransfected human GCK and GKRP in HeLa cells at $5.5 \mathrm{mmol} / \mathrm{l}$ glucose. a Visualisation of human EGFP-GCK and DAPI. b,c Co-transfection of mCherry-WT-hGKRP and EGFP-GCK (b) or mCherryP446L-hGKRP and EGFP-GCK (c): GKRP signal, upper left; GCK signal, upper right; DAPI, lower left; overlay, lower right. d Quantification of degree of nuclear fluorescence of GKRP and GCK. Data are expressed as means \pm SEM of two individual experiments with a total of $77-$ 98 cells analysed. ${ }^{*} p<0.05$; $* * * p<0.001$ ( $t$ test). Black bars, GKRP; white bars, GCK

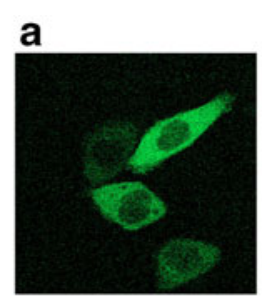

b

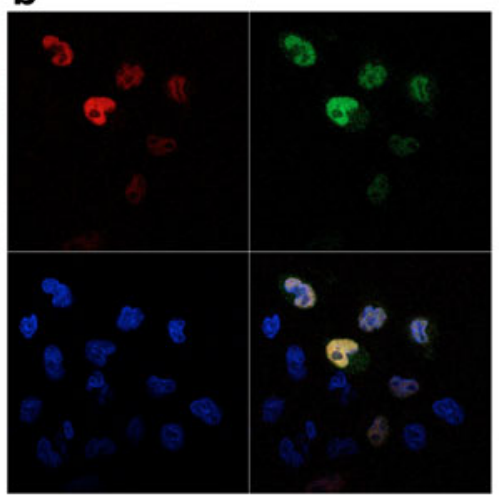

C

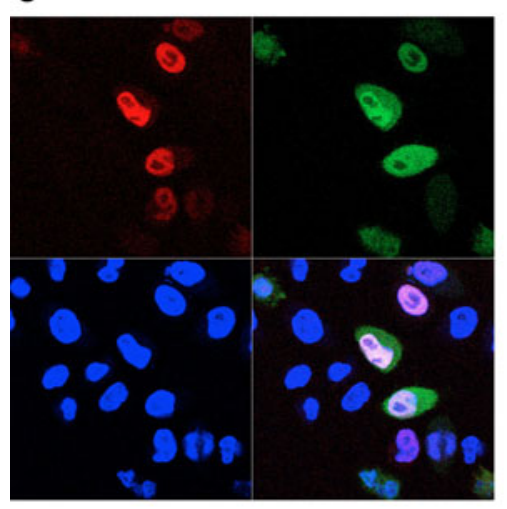

d

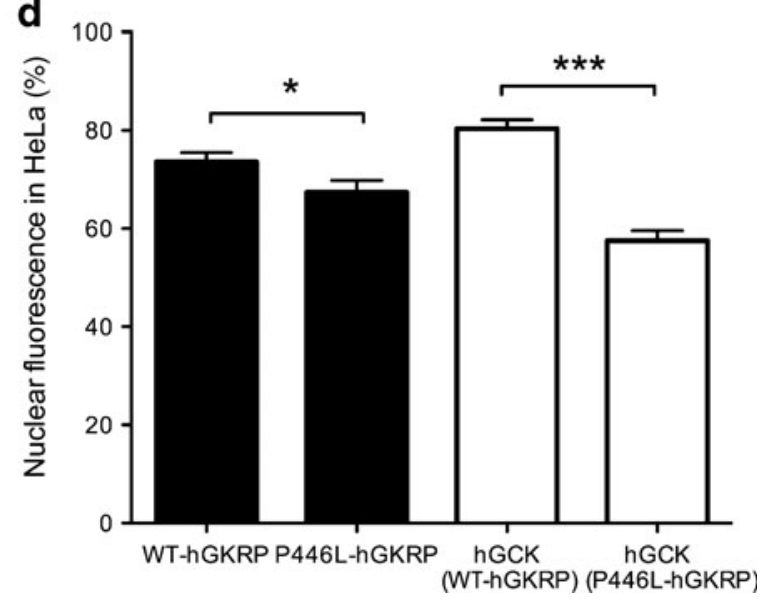

nuclear fluorescence; $p=0.04)$. Human GCK showed significantly decreased nuclear localisation in the presence of P446L-hGKRP (Fig. 2d; $57.5 \pm 2.1 \%$ vs $80.4 \pm 1.8 \%$ nuclear fluorescence; $p<0.001$ ). The nuclear localisation of GCK remained significantly reduced in the presence of P446LhGKRP compared with WT-hGKRP when individual cells were matched for mCherry, EGFP or mCherry:EGFP fluorescence intensity ( $p<0.05$ for all groups).

There was no significant difference in GCK sequestration between high and low glucose for either human variant (Fig. 3). The differences in GCK sequestration between WThGKRP and P446L-hGKRP observed at $5.5 \mathrm{mmol} / \mathrm{l}$ glucose were also detectable at $25 \mathrm{mmol} / \mathrm{l}$ glucose $(p<0.001$ for GKRP and GCK localisation). By comparison, transient cotransfection of rGKRP proteins with human GCK resulted in no difference in GCK sequestration between WT-rGKRP and P446L-rGKRP ( $p>0.1$ for both glucose concentrations). However, there was a significant glucose dependence in GCK sequestration by both rGKRPs, with increased GCK nuclear localisation observed at low glucose (Fig. 3; $p<0.05$ for both WT-rGKRP and P446L-rGKRP). These findings are not likely due to differences in rat and human GCK, as previous kinetic studies have extensively characterised the interaction between rat and human GKRP and either rat or human GCK, showing no difference in interaction of GKRP with either rat or human GCK [14].

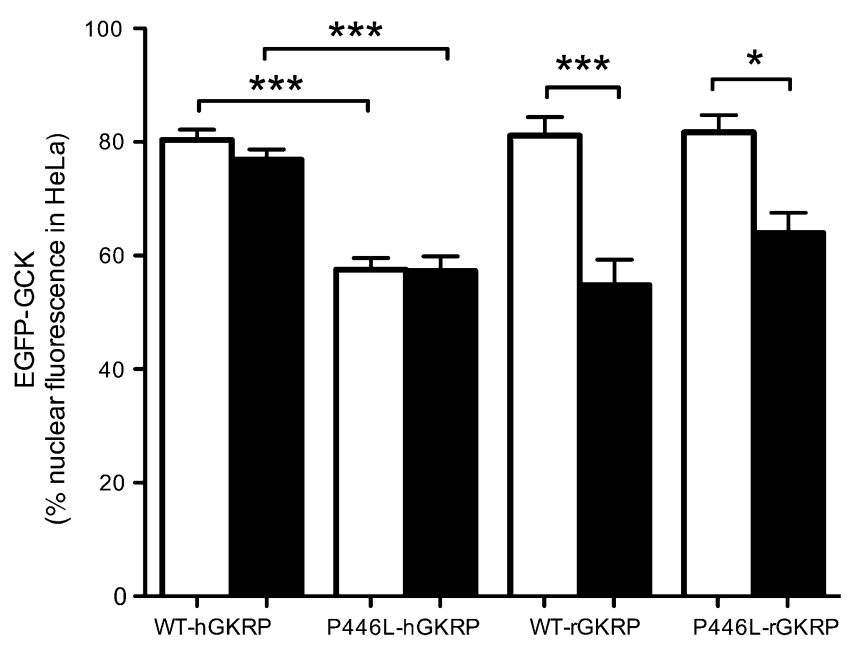

Fig. 3 Glucose dependence of GCK localisation in HeLa cells. Degree of nuclear localisation of human GCK transiently transfected in the presence of WT-hGKRP, P446L-hGKRP, WT-rGKRP or P446L-rGKRP at $5.5 \mathrm{mmol} / 1$ and $25 \mathrm{mmol} / 1$ glucose. Data are expressed as means \pm SEM of two individual experiments with a total of $18-98$ cells analysed. ${ }^{*} p<0.05 ; * * *<0.001$ (ANOVA/Bonferroni correction). White bars, $5.5 \mathrm{mmol} / \mathrm{l}$ glucose; black bars, $25 \mathrm{mmol} / \mathrm{l}$ glucose 
Direct interaction of human GCK and GKRP in HeLa cells FRETN was used to detect the interaction between ECFP-GCK and EYFP, EYFP-WT-hGKRP, or EYFPP446L-hGKRP in the nucleus of HeLa cells. FRETN measures the transfer of energy from ECFP to EYFP on excitation of ECFP and occurs when these proteins are within close proximity $(<10 \mathrm{~nm})$ because of the overlap of the emission spectrum of ECFP and the excitation spectrum of EYFP [22]. There was a significant reduction in interaction with GCK for the P446L-hGKRP variant protein compared with WT-hGKRP at $5.5 \mathrm{mmol} / \mathrm{l}$ glucose (Fig. 4). WT-hGKRP showed significantly reduced interaction with GCK at $25 \mathrm{mmol} / 1$ glucose compared with $5.5 \mathrm{mmol} / \mathrm{l}$. There was no significant glucose dependence of interaction for P446L-hGKRP.

Direct interaction and cellular localisation of human GCK and GKRP in primary mouse hepatocytes To compare these results with those with primary cells, we assessed the localisation and interaction of transiently transfected human GCK and hGKRP in mouse hepatocytes. The importance of endogenous mouse GCK and GKRP in this cell type was emphasised by our observation that transfected ECFP-GCK localises strongly to the nucleus at $5.5 \mathrm{mmol} / \mathrm{l}$ glucose and translocates to the cytoplasm in a glucose-dependent manner in the absence of transfected hGKRP (Fig. 5a). Translocation of ECFP-GCK was not abolished by the presence of EYFP-WT-hGKRP. However, EYFP-P446LhGKRP abolished glucose-dependent translocation of

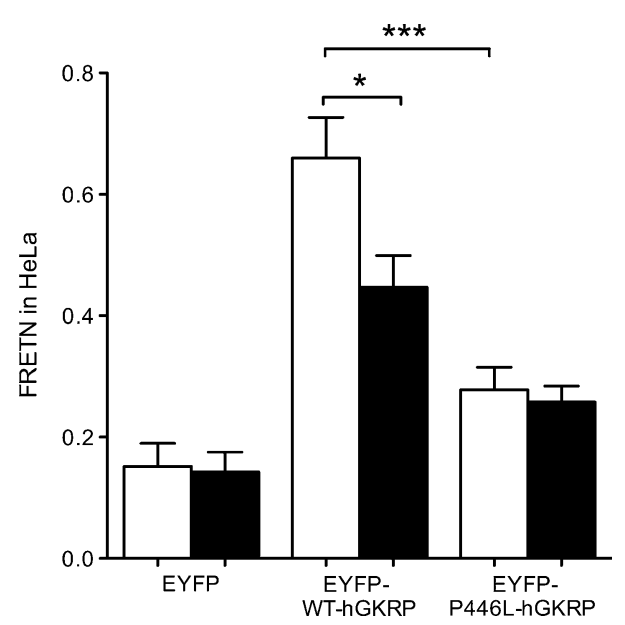

Fig. 4 Direct interaction between human GCK and WT-hGKRP or P446L-hGKRP in HeLa cells. HeLa cells were transiently transfected with plasmids encoding ECFP-GCK and EYFP, EYFP-WT-hGKRP or EYFP-P446L-hGKRP as indicated and cultured in medium with 5.5 or $25 \mathrm{mmol} / \mathrm{l}$ glucose. Thereafter, fluorescence images were taken in living cells and the FRETN was calculated from the ECFP and EYFP emission intensities in the nucleus. Data are expressed as means \pm SEM of two individual experiments with a total of 7-10 cells analysed; ${ }^{*} p<0.05 ; * * * p<0.001$ (ANOVA/Bonferroni correction). White bars, $5.5 \mathrm{mmol} / 1$ glucose; black bars, $25 \mathrm{mmol} / 1$ glucose
ECFP-GCK. This may be due to an abundance of EYFPP446L-hGKRP protein in the cytoplasm preventing GCK from localising to the nucleus even at low glucose concentrations, as suggested by our assessment of GCK localisation in HeLa cells (Fig. 3). As in HeLa cells, we observed significantly decreased interaction between GCK and P446LhGKRP compared with WT-hGKRP at $5.5 \mathrm{mmol} / 1$ glucose in hepatocytes (Fig. 5b). At $25 \mathrm{mmol} / \mathrm{l}$ glucose in hepatocytes, accelerated metabolism results in generation of high levels of $\mathrm{NADH}$ and NADPH, resulting in autofluorescence. Additionally, there is a reduction in the absolute value of FRETN compared with HeLa cells due to quenching by endogenous mouse GCK and GKRP. Accordingly, the reduced interaction between GCK and GKRP at $25 \mathrm{mmol} / \mathrm{l}$ glucose was not significantly detectable above this increased background (Fig. 5b).

\section{Discussion}

To capitalise on recent advances in our understanding of the genetic basis of diabetes risk, it is necessary to translate association signals into detailed molecular mechanisms that may underlie disease predisposition. We have previously shown that P446L-hGKRP, inversely associated with triacylglycerol levels and glucose levels, affects GCK activity in liver through diminished regulation by F6P [12]. However, the impact of this variant on the cellular localisation of GCK and GKRP has not previously been explored. Recent studies using rat hepatocytes have suggested reliable estimation of GCK localisation can be accomplished by quantification of localisation in multiple fields of cells $[25,26]$. We therefore sought to image a large number of cells using a quantitative method to assess nuclear and cytoplasmic fluorescence, and to combine this with assessment of direct interaction of GKRP and GCK by measuring FRETN. We have demonstrated for the first time differences in the degree of nuclear sequestration of GCK between WT-hGKRP and P446L-hGKRP and in the direct cellular interaction of P446L-hGKRP with GCK compared with WT-hGKRP. In spite of the presence of endogenous mouse GCK and GKRP, we were also able to demonstrate these differences in localisation, sequestration and direct interaction in primary mouse hepatocytes.

In combination with our previously described kinetic studies, the P446L-hGKRP variant protein would be expected to result in an increased pool of active cytoplasmic GCK, particularly in the presence of low (i.e. fasting state) glucose concentrations. Our findings suggest a mechanism of action for P446L-hGKRP consistent with the associated clinical phenotypes [9-11]. The reduced ability of P446L-hGKRP to sequester and interact with 

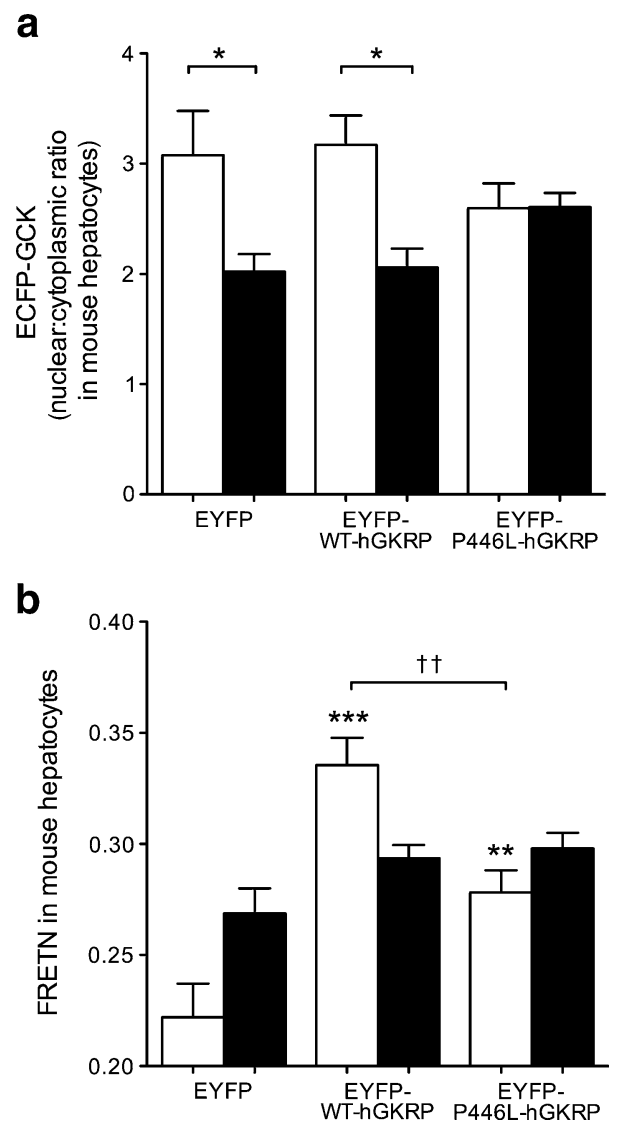

Fig. 5 Localisation of human GCK and direct interaction with WThGKRP or P446L-hGKRP in primary mouse hepatocytes. Hepatocytes were transiently transfected with plasmids encoding ECFP-GCK and EYFP, EYFP-WT-hGKRP or EYFP-P446L-hGKRP as indicated and cultured in medium with 5.5 or $25 \mathrm{mmol} / 1$ glucose. Thereafter, fluorescence images were taken in living cells. a Calculation of the ECFP-GCK nuclear:cytoplasmic ratio. Data are expressed as means \pm SEM of two individual experiments with a total of 9-12 cells analysed; $* p<0.05$ (ANOVA/Bonferroni correction). b Calculation of FRETN from the ECFP and EYFP emission intensities in the nucleus and cytoplasm. Data are expressed as means \pm SEM of two individual experiments with a total of $6-7$ cells analysed; $* * p<0.01$; $* * * p<0.001$ compared with control; ${ }^{\dagger \dagger} p<0.01$ compared with WT (ANOVA/Bonferroni correction). White bars, $5.5 \mathrm{mmol} / \mathrm{l}$ glucose; black bars, $25 \mathrm{mmol} / 1$ glucose

GCK in the nucleus in the fasting state would result in increased hepatic glucose disposal, glycolytic flux and generation of precursors for synthesis of molecules such as triacylglycerols and cholesterol. Recent findings associating GCKR with increased VLDL particle concentrations [27] suggest this enhancement of glycolytic flux leads to increased levels of de novo triacylglycerol and cholesterol synthesis and export.

Increased fasting-state hepatic glucose uptake, use and disposal by GCK in the presence of P446L-hGKRP would be predicted to decrease circulating plasma glucose concentrations. Further support for this molecular mechanism comes from at least one cellular study linking over- expression of $G C K$ in liver to reduced glucose levels and raised triacylglycerol levels [28]. We propose that decreased fasting plasma glucose levels could result in decreased basal insulin output by the pancreas, which could ultimately contribute to higher insulin sensitivity and decreased risk of type 2 diabetes in spite of increased hepatic lipid production.

Our results also suggest that, compared with WThGKRP, P446L-hGKRP shows reduced glucose dependence in its cellular interaction with GCK (Fig. 4). Accordingly, in spite of higher fasting-state GCK activity in the presence of P446L-hGKRP, GCK may not be activated to the same extent by increased glucose in the presence of this variant. This provides a mechanistic explanation for the observation that the $\mathrm{P} 446 \mathrm{~L}$ variant is associated with increased $2 \mathrm{~h}$ glucose levels but decreased fasting glucose levels [10, 29].

Interestingly, the degree of nuclear localisation of rGKRP is significantly reduced compared with WT-hGKRP in both HeLa cells and hepatocytes. Our studies assessing the localisation of transfected rGKRP in mouse hepatocytes (nuclear/cytoplasmic ratio of 3.37 at $5.5 \mathrm{mmol} / \mathrm{l}$ glucose) are highly consistent with our observations of endogenous rGKRP in rat hepatocytes (nuclear/cytoplasmic ratio of 3.44 at $5.5 \mathrm{mmol} / \mathrm{l}$ glucose; S. Baltrusch, unpublished observations). However, the abnormality in nuclear GCK sequestration for P446L-GKRP appears to be specific to the human regulatory protein as we did not observe a similar phenomenon with rGKRP. This is consistent with previous studies that have established important speciesspecific differences in both the regulation of human and rat GKRP by F6P and the impact of the P446L variant on this regulation [12-14]. Taking into account our previous assessment of the interaction of rGKRP with human GCK by FRETN analysis in COS-1 cells [21], direct interaction determined in our experiments in living cells appeared to be strongest between hGKRP and GCK. This is consistent with in vitro studies highlighting hGKRP as a more potent inhibitor of GCK than rGKRP [14]. These studies serve to emphasise the differences between human and rat regulatory proteins and highlight the need for use of appropriate assays to decipher complex mutational mechanisms driving disease associations.

It has previously been reported that glucose-dependent GCK localisation is highly heterogeneous in rat hepatocytes [25], thus necessitating careful analysis in living and fixed cells. Our results comparing the glucose dependence of the nuclear/cytoplasmic ratio for human GCK co-transfected with rGKRP in mouse hepatocytes resulted in a ratio at $25 \mathrm{mmol} / \mathrm{l}$ that was $63 \%$ of the ratio at $5.5 \mathrm{mmol} / 1$ glucose. This is consistent with the ratio calculated from the results of Watanabe et al. [25] analysing endogenous rat GCK $(59 \%)$ and our previous results measuring the nuclear/ cytoplasmic ratio of transfected human GCK in rat 
hepatocytes (61\%) [17]. Translocation of human GCK in the presence of hGKRP has not been previously explored, but our results suggest there are important differences between the rat and human GKRP translocation systems, and that regulation of translocation in humans will be strongly dependent on the genotype at P446L. Extensive assessment in human hepatocytes of appropriate genotype may provide useful future insight into these questions.

In conclusion, we have demonstrated that the human GCKR P446L variant alters the ability of GKRP to sequester GCK in the nucleus. This observation, coupled with our previous kinetic studies, provides a mechanistic link between a common genetic variant associated with diabetes risk and metabolic traits and glucose and triacylglycerol metabolism.

Acknowledgements This study was supported by the NIH Division of Intramural Research and NHGRI project number Z01-HG000024 (F.S. Collins). This study was funded in Oxford by the Medical Research Council (81696) and the Wellcome Trust (095101/Z/10/Z). A.L. Gloyn is a Wellcome Trust Senior Research Fellow in Basic and Biomedical Science. This study was funded in Rostock by the German Diabetes Association (DDG).

Contribution statement MGR wrote the manuscript, contributed to experimental design, and researched and analysed data. SW analysed data and contributed to the manuscript. JS researched and analysed data and contributed to the manuscript. RW researched and analysed data and contributed to the manuscript. NLB researched and analysed data and reviewed and edited the manuscript. SB researched and analysed data and reviewed and edited the manuscript. FSC contributed to experimental design and reviewed and edited the manuscript. ALG contributed to experimental design and wrote the manuscript. All authors approved the final version to be published.

Duality of interest The authors declare that there is no duality of interest associated with this manuscript.

\section{References}

1. McCarthy MI (2010) Genomics, type 2 diabetes, and obesity. New Engl J Med 363:2339-2350

2. van de Bunt M, Gloyn AL (2010) From genetic association to molecular mechanism. Curr Diab Rep 10:452-466

3. Hindorff LA, Sethupathy P, Junkins HA et al (2009) Potential etiologic and functional implications of genome-wide association loci for human diseases and traits. Proc Natl Acad Sci USA 106:9362-9367

4. Van Schaftingen E (1989) A protein from rat liver confers to glucokinase the property of being antagonistically regulated by fructose 6-phosphate and fructose 1-phosphate. Eur J Biochem 179:179-184

5. Vandercammen A, Detheux M, van Schaftingen E (1992) Binding of sorbitol 6-phosphate and of fructose 1-phosphate to the regulatory protein of liver glucokinase. Biochem J 286:253-256

6. Toyoda Y, Miwa I, Satake S, Anai M, Oka Y (1995) Nuclear location of the regulatory protein of glucokinase in rat liver and translocation of the regulator to the cytoplasm in response to high glucose. Biochem Biophys Res Commun 215:467-473

7. Shiota C, Coffey J, Grimsby J, Grippo JF, Magnuson MA (1999) Nuclear import of hepatic glucokinase depends upon glucokinase regulatory protein, whereas export is due to a nuclear export signal sequence in glucokinase. J Biol Chem 274:37125-37130

8. Kaminski MT, Lenzen S, Baltrusch S (2010) New aspects of hepatic glucose phosphorylation by intracellular glucokinase localisation. Diabetologia 53:(Suppl 1). Abstract 629

9. Orho-Melander M, Melander O, Guiducci C et al (2008) Common missense variant in the glucokinase regulatory protein gene is associated with increased plasma triglyceride and C-reactive protein but lower fasting glucose concentrations. Diabetes 57:3112-3121

10. Dupuis J, Langenberg C, Prokopenko I et al (2010) New genetic loci implicated in fasting glucose homeostasis and their impact on type 2 diabetes risk. Nat Genet 42:105-116

11. Teslovich TM, Musunuru K, Smith AV et al (2010) Biological, clinical and population relevance of 95 loci for blood lipids. Nature 466:707-713

12. Beer NL, Tribble ND, McCulloch LJ et al (2009) The P446L variant in GCKR associated with fasting plasma glucose and triglyceride levels exerts its effect through increased glucokinase activity in liver. Hum Mol Genet 18:4081-4088

13. Veiga-da-Cunha M, Delplanque J, Gillain A et al (2003) Mutations in the glucokinase regulatory protein gene in 2 p23 in obese French Caucasians. Diabetologia 46:704-711

14. Brocklehurst KJ, Davies RA, Agius L (2004) Differences in regulatory properties between human and rat glucokinase regulatory protein. Biochem J 378:693-697

15. Farrelly D, Brown KS, Tieman A et al (1999) Mice mutant for glucokinase regulatory protein exhibit decreased liver glucokinase: a sequestration mechanism in metabolic regulation. Proc Natl Acad Sci USA 96:14511-14516

16. Grimsby J, Coffey JW, Dvorozniak MT et al (2000) Characterization of glucokinase regulatory protein-deficient mice. J Biol Chem 275:7826-7831

17. Baltrusch S, Francini F, Lenzen S, Tiedge M (2005) Interaction of glucokinase with the liver regulatory protein is conferred by leucine-asparagine motifs of the enzyme. Diabetes 54:28292837

18. Shaner NC, Campbell RE, Steinbach PA, Giepmans BN, Palmer $\mathrm{AE}$, Tsien RY (2004) Improved monomeric red, orange and yellow fluorescent proteins derived from Discosoma sp. red fluorescent protein. Nat Biotechnol 22:1567-1572

19. Seglen PO (1976) Preparation of isolated rat liver cells. Methods Cell Biol 13:29-83

20. Bader A, Fruhauf N, Tiedge M et al (1999) Enhanced oxygen delivery reverses anaerobic metabolic states in prolonged sandwich rat hepatocyte culture. Exp Cell Res 246:221-232

21. Langer S, Kaminski MT, Lenzen S, Baltrusch S (2010) Endogenous activation of glucokinase by 6-phosphofructo-2-kinase/ fructose-2,6-bisphosphatase is glucose dependent. Mol Endocrinol 24:1988-1997

22. Vanderklish PW, Krushel LA, Holst BH, Gally JA, Crossin KL, Edelman GM (2000) Marking synaptic activity in dendritic spines with a calpain substrate exhibiting fluorescence resonance energy transfer. Proc Natl Acad Sci USA 97:2253-2258

23. Rodriguez-Enriquez S, Marin-Hernandez A, Gallardo-Perez JC, Moreno-Sanchez R (2009) Kinetics of transport and phosphorylation of glucose in cancer cells. J Cell Physiol 221:552-559

24. Mukhtar M, Stubbs M, Agius L (1999) Evidence for glucose and sorbitol-induced nuclear export of glucokinase regulatory protein in hepatocytes. FEBS Lett 462:453-458

25. Watanabe F, Furuya E (2010) Quantitative image analysis reveals that phosphorylation of liver-type isozyme of fructose-6phosphate 2-kinase/fructose-2,6-bisphosphatase does not affect 
nuclear translocation of glucokinase in rat primary hepatocytes. J Biochem Dec 148:713-719

26. Wolff M, Kauschke SG, Schmidt S, Heilker R (2008) Activation and translocation of glucokinase in rat primary hepatocytes monitored by high content image analysis. J Biomol Screen 13:837-846

27. Stancakova A, Paananen J, Soininen P et al (2011) Effects of 34 risk loci for type 2 diabetes or hyperglycemia on lipoprotein subclasses and their composition in 6,580 nondiabetic Finnish men. Diabetes 60:1608-1616

28. Slosberg ED, Desai UJ, Fanelli B et al (2001) Treatment of type 2 diabetes by adenoviral-mediated overexpression of the glucokinase regulatory protein. Diabetes 50:1813-1820

29. Saxena R, Hivert MF, Langenberg $C$ et al (2010) Genetic variation in GIPR influences the glucose and insulin responses to an oral glucose challenge. Nat Genet 42:142-148 